\title{
Automatic segmentation of relevant textures in agricultural images
}

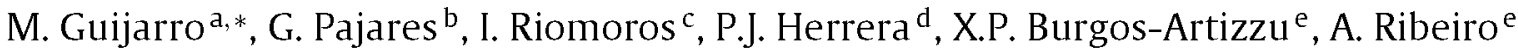 \\ a Centro de Estudios Superiores Felipe II.- Aramjuez, Facultad Informática, Universidad Complutense, 28040 Madrid, Spain \\ ${ }^{\mathrm{b}}$ Dpto. Ingeniería del Software e Inteligencia Artificial, Facultad Informática, Universidad Complutense, 28040 Madrid, Spain \\ ${ }^{c}$ Dpto. Sistemas Informáticos y Programación, Facultad Informática, Universidad Complutense, 28040 Madrid, Spain \\ ${ }^{d}$ Dp to. Arquitectura Computadores y Automática, Facultad Informática, Universidad Complutense, 28040 Madrid, Spain \\ e Grupo de Percepción Artificial, Instituto de Automática Industrial, Consejo Superior de Investigaciones Científicas, Arganda del Rey, Madrid, Spain
}

Keywords:

Machine vision

Image segmentation

Texture identification in crops

Automatic tasks in agriculture

\begin{abstract}
A B S T R A C T
One important issue emerging strongly in agriculture is related with the automatization of tasks, where the optical sensors play an important role. They provide images that must be conveniently processed. The most relevant image processing procedures require the identification of green plants, in our experiments they come from barley and corn crops including weeds, so that some types of action can be carried out, including site-specific treatments with chemical products or mechanical manipulations. Also the identification of textures belonging to the soil could be useful to know some variables, such as humidity, smoothness or any others. Finally, from the point of view of the autonomous robot navigation, where the robot is equipped with the imaging system, some times it is convenient to know not only the soil information and the plants growing in the soil but also additional information supplied by global references based on specific areas. This implies that the images to be processed contain textures of three main types to be identified: green plants, soil and sky if any. This paper proposes a new automatic approach for segmenting these main textures and also to refine the identification of sub-textures inside the main ones. Concerning the green identification, we propose a new approach that exploits the performance of existing strategies by combining them. The combination takes into account the relevance of the information provided by each strategy based on the intensity variability. This makes an important contribution. The combination of thresholding approaches, for segmenting the soil and the sky, makes the second contribution; finally the adjusting of the supervised fuzzy clustering approach for identifying sub-textures automatically, makes the third finding. The performance of the method allows to verify its viability for automatic tasks in agriculture based on image processing.
\end{abstract}

(c) 2010 Elsevier B.V. All rights reserved.

\section{Introduction}

\subsection{Problem statement}

The increasing development of robotics equipped with machine vision sensors applied to Precision Agriculture (PA) is demanding solutions for several problems. The robot navigates and acts over a site-specific area of a larger farm (Davies et al., 1998), where one important part of the information is supplied by the vision system.

The main issue related with the application of machine vision methods is that concerning the image segmentation. Efficient and automatic segmentation of vegetation from images of the ground is an important step for many applications such as weed detection for site-specific treatment (Onyango and Marchant, 2003; Tellaeche et al., 2008a,b; Burgos-Artizzu et al., 2009). Also the ground classification covers a number of ecologically relevant categories (Luscier et al., 2006).

Normally, the autonomous navigation requires global information in the image to fix reference points and the action needs the identification of specific and relevant areas. This implies that the image segmentation process must deal with the full image globally and simultaneously must be able to capture details of a specific area for identifying textures of plants and soil. The spectral signatures of cereal, corn and weeds are very similar. Moreover, because the management is carried out in hostile outdoor environments, several factors and adverse conditions are to be considered, such as shadows or different spectral signatures for the same type of plants depending on the illumination, for example, consider the robot navigating with a view to the sun and then of backs to the sun. Also variations in illumination due to climatic conditions could cause important changes in the spectral components (Tian and Slaughter, 1998), consider a day with high density of clouds occluding the sun continuously. In this paper, we design a new strategy that can cope with this. 


\subsection{Revision of methods}

Several strategies have been proposed for segmenting crop canopy images, specifically oriented towards green segmentation:

(1) Visible spectral-index based, including the excess green index (ExG, Woebbecke et al., 1995; Ribeiro et al., 2005), the excess red index (ExR, Meyer et al., 1998), the colour index of vegetation extraction (CIVE, Kataoka et al., 2003), and the excess green minus excess red index (ExGR, Neto, 2004). And the vegetative index (VEG) described in Hague et al. (2006), which was designed to cope with the variability of natural daylight illumination. All these approaches need to fix a threshold for final segmentation.

(2) Specific threshold-based approaches, including dynamic thresholding. Generally, these techniques assume a two-class problem where plants and soil are to be identified. Reid and Searcy (1987) estimated a decision function under the assumption that the classes follow Gaussian distributions. The Otsu's method (Otsu, 1979) was also applied considering a bi-class problem (Ling and Ruzhitsky, 1996; Shrestha et al., 2004). These algorithms are applied to gray images. Gebhardt et al. (2006) applied also thresholding for segmentation transforming the images from RGB to gray scale intensity. This algorithm was later improved using local homogeneity and morphological operations in Gebhardt and Kaühbauch (2007). Kirk et al. (2009) applied a combination of greenness and intensity derived from the red and green spectral bands and compute an automatic threshold for a two-class problem assuming two Gaussian probability density functions associated to soil and vegetation respectively; this procedure requires the previous estimation of an angle to rotate the hypothetical greenness axis. Meyer and Camargo-Neto (2008) applied the automatic Otsu's thresholding method for binarizing ExG and the normalized difference index (NDI), where a comparison was established against the segmentation obtained from ExGR determining that in this last case, a value of zero suffices for the threshold, therefore the Otsu's method is not required.

(3) Learning-based, Meyer et al. (2004) applied unsupervised approaches, including fuzzy clustering, for segmenting regions of interest from ExR and ExG. Tian and Slaughter (1998) proposed the environmentally adaptive segmentation algorithm (EASA) for detecting plants through a supervised learning process. Ruiz-Ruiz et al. (2009) applied the EASA later under the HSI (hue-saturationintensity) color space to deal with the illumination variability. Zheng et al. (2009, 2010) used a supervised mean-shift algorithm under the assumption that the segmentation of green vegetation from a background can be treated as a two-class segmentation problem; the class separability was validated through a neural network and the Fisher linear discriminant respectively, the color spaces used were RGB, LUV and HSI.

Our problem is the segmentation of images containing barley and corn crops. Moreover, the vision system is mounted on a vehicle, which navigates in the field. Two types of images can be obtained: (1) those by focusing the ground and containing only both green plants and soil, Fig. 1; (2) those obtained by focusing the horizon, which are suitable for navigation purposes because of the panoramic perspective provided, they contain three main classes, Fig. 2: green vegetation, soil and also part of the sky. This last means that bi-class learning-based approaches are not suitable in our application. Therefore, we must design our automatic strategy to cope with these two types of images and also consider that the images could be acquired under different illumination conditions. This means that the method should be robust enough in images rich in illumination, Fig. 2, where the images were captured in a sunny stable day or with poor illumination, Fig. 3, where the images were obtained perhaps at late afternoon or in a day where the illumination was changing continuously.

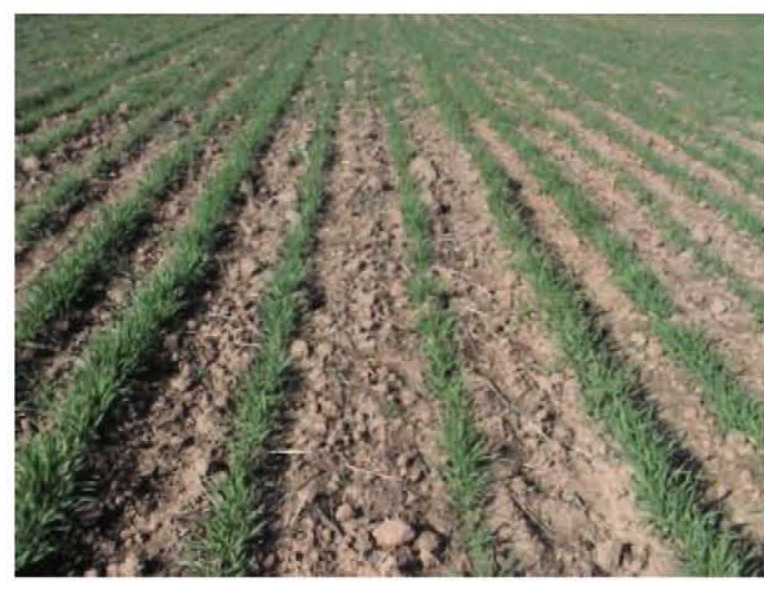

Fig. 1. Original image displaying only ground.

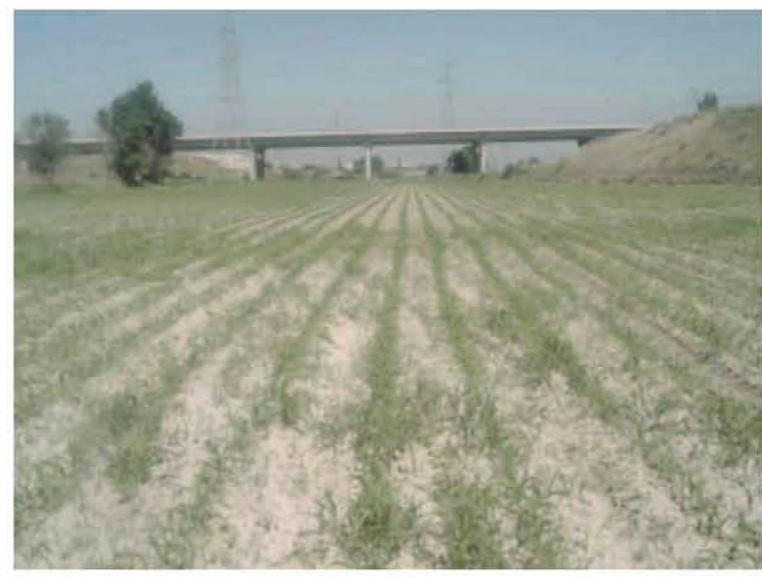

Fig. 2. Original image displaying ground and sky highly illuminated.

\subsection{Motivational research and design of the proposed strategy}

Based on the aforementioned considerations, we revised these strategies from our point of view. As reported in Meyer and Camargo-Neto (2008) the advantage of using color indices is that they accentuate a particular color, which is of interest. The images analyzed contain three main dominant spectral signatures, green for plants, red for soil and blue for sky; therefore we might think to use greenness indices for the first and redness for soil identi-

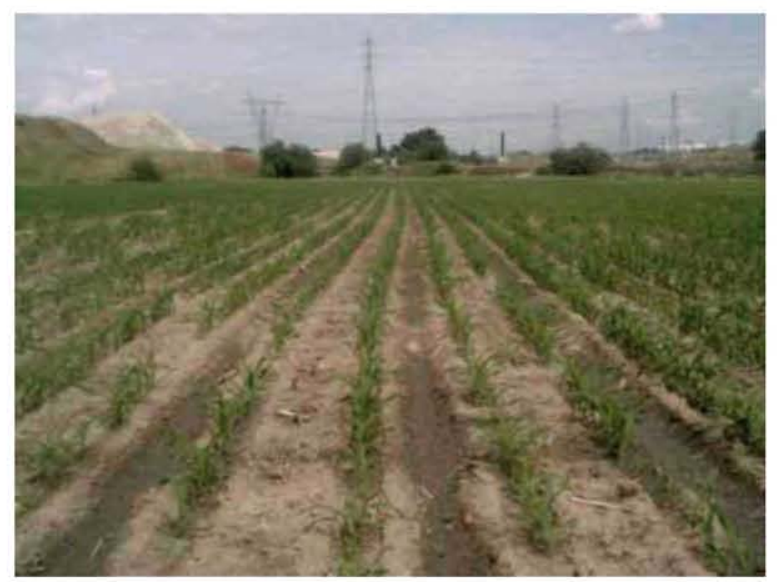

Fig. 3. Original image displaying ground and sky captured at dusk with poor illumination. 
fication. With such purpose, we selected ExG, CIVE, ExGR and VEG for greenness and ExR for redness, because of their apparent good results, unlike NDI that identifies a lot of pixels in the sky as green ones. The blueness has not been considered in the literature, but we proposed a simple translation from redness by changing the red spectral component by the blue one. This allows detecting specific signatures belonging to the sky with a certain degree of robustness, where the resulting images are called ExB. Inspired on Meyer and Camargo-Neto (2008), we also applied an automatic thresholding approach, but instead of using the Otsu's method we apply as threshold the mean value of the gray intensity images. This is because, in our images, we verified that the threshold obtained through the Otsu's method is always greater than the mean and in most cases produce infra-segmentation, this means that there are pixels belonging to a green textures that are excluded, as we will explain later.

We verified, in the set of images analyzed, that the methods based on ExG, CIVE, ExGR and VEG, do not work in the same way; depending on the images some of them produce over-segmentation (excessive green is extracted) or under-segmentation (little green), without any apparent reason, normally not all simultaneously in the same sense. This fact motivated the design of the proposed automatic combined approach making use of the information provided by ExG, CIVE, ExGR and VEG. Now the problem is to choose the combination scheme. Because they contain all greenness information and it is accentuated on the green spectral band, the plants in the images appear with intensity levels greater than the remainder and moreover, normally the plants in the image display high contrast variability due to different degrees of greenness displayed by crops and weeds and also due to the shadows, which vary depending on the growth state of the plants. There are several techniques that can capture this variability such as entropy or uniformity (Gonzalez and Woods, 2008), as we will see later both work similarly and the uniformity is selected because of its simplicity. Under these considerations we extracted the greenness. The redness was obtained directly by the computation of the ExR intensity image. The blueness was also obtained as mentioned before in ExB. The design of an appropriate procedure, described in Section 2.3.1 allows us to separate the three main textures, i.e. green, sky and soil.

But still it is possible to improve the segmentation procedure in two ways. Considering the pixels classified as green, we applied a fuzzy classifier for identifying different types of greenness, probably coming from different types of plants, such as crop and weeds or shadows in the plants. Moreover in some images, mainly coming from corn crops, with low densities of plants there are some of them which are identified erroneously as soil because of its spectral components, with domain of the red one against the green. This does not represent a failure of the greenness procedure but it is a problem derived from the spectral signature due to the acquisition process, including the design of the devices. Based on the proposed approach, we can still identify some plants which were erroneously classified as soil. This was achieved by applying a fuzzy classifier, as above.

In summary the proposed automatic image segmentation approach consists of the following three main steps, which are later described in depth:

1. Extract the greenness by combining ExG, CIVE, ExGR and VEG based on the uniformity of the corresponding histograms.

2. Extract the redness through ExR and also the blueness by applying the same criterion for the blue band. Identify if the image contains sky or only soil and plants.

3. Classify different types of plants and soil based on a fuzzy clustering approach.

\section{Materials and methods}

\subsection{Images}

The images used for this study belongs to two types of crops barley and corn. The first ones were captured with a Sony DCR PC110E camera during April 2006 in a 1.7-ha experimental field of barley on La Poveda Research Station, Arganda del Rey, Madrid. The second ones were acquired with a HPR817 digital in four different days in April/May 2007. All acquisitions were spaced by five/six days, i.e. they were obtained under different conditions of illumination and different growth stages. The digital images were captured under perspective projection and stored as 24-bit color images with resolutions of $1152 \times 864$ pixels and $800 \times 600$ respectively and saved in RGB (Red, Green and Blue) color space in the JPG format. The images were processed with the Image Processing Toolbox from Matlab R2009a (MathWorks, 2010). A set of 240 images were processed.

Fig. 1 is an original image belonging to a field of barley (first type); only the ground was captured under a high quality in the illumination. Figs. 2 and 3 belong to a field of corn (second type), where ground and sky textures were captured, but under different illumination conditions (high and low contrast respectively); Fig. 3 was obtained at dusk, i.e. with poor illumination. These three images are representative examples of the set of images processed.

\subsection{The automatic segmentation method}

The proposed strategy is based on the following process, according to the three main steps introduced in Section 1.3:

(1) Color space normalization: given an original input image in the RGB color space, we applied the following normalization scheme, which is usually applied in agronomic image segmentation (Gée et al., 2008), obtaining the normalized spectral $r, g$ and $b$ components ranging in $[0,1]$ according to (1),

$r=\frac{R}{R+G+B}, \quad g=\frac{G}{R+G+B}, \quad b=\frac{B}{R+G+B}$

where $R, G$ and $B$ are the normalized RGB coordinates ranging from 0 to 1 and are obtained as follows:

$R=\frac{R}{R_{\max }}, \quad G=\frac{G}{G_{\max }}, \quad B=\frac{B}{B_{\max }}$

where $R_{\max }=G_{\max }=B_{\max }=255$ for our 24-bit color images.

(2) Indices computation: they are computed as follows (see references above in Section 1.2),

Excess green: $E \times G=2 g-r-b$

Excess red : $E x R=1.4 r-g$

Excess blue: $\operatorname{ExB}=1.4 b-\mathrm{g}$

Excess green minus excess red: $E x G R=E x G-E x R$

Color index of vegetation extraction:

CIVE $=0.441 r-0.811 g+0.385 b+18.78745$

Vegetative: $\quad V E G=\frac{g}{r^{a} b^{1-a}}$ with $a$ set to 0.667

as in Hague et al. (2006)

(3) Combination of green indices: the starting points are the indices specifically oriented towards the determination of the greenness, i.e. ExG, ExGR, CIVE and VEG. The goal is to fuse the four images by combining them. To illustrate the fusion procedure, consider Fig. 4(a) and (b) where the greenness corresponding to the images EXG and ExGR respectively are displayed. They are obtained 

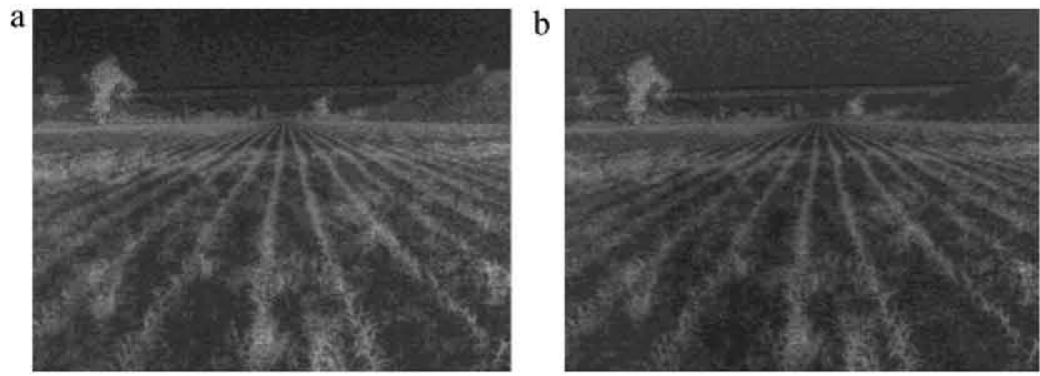

Fig. 4. Different degrees of greenness from image in Fig. 2 (a) ExG and (b) ExGR.

by applying Eqs. (3) and (6) to the original image in Fig. 2. As we can see, the plants appear with gray levels greater than the remainder parts in the image (soil and sky), although both images appear to be very similar, they really contain different intensity levels among them. Obviously, as the contrast increases, the discrimination improves. Therefore, the image with the greatest contrast will contribute more in the resulting combined image. The contrast concept can be measured based on the gray-level histogram, we have two available measures either uniformity or entropy (Gonzalez et al., 2004; Gonzalez and Woods, 2008). Uniformity is maximum when all gray levels are equal (maximally uniform) and decreases from there. Entropy is a measure of randomness or variability, which is minimum when all gray levels are equal. Therefore, we searched for minimum uniformity and maximum entropy, because they provide a high discriminatory power. We verified that both measures have a similar behavior in our set of images; therefore, we chose the easiest from the computational point of view, i.e. the uniformity, which is computed as follows:

$U=\sum_{i=0}^{L-1} p^{2}\left(z_{i}\right)$

where $z$ is a random variable denoting gray levels and $p\left(z_{i}\right)$ the histogram, $i=0,1,2, \ldots, L-1$, where $L$ is the number of distinct gray levels. Because, our original image representation is 8 -bits in gray level, the maximum value is $2^{8}=256$, i.e. $L=256$. Our normalized gray levels values vary in the continuous interval $[0,1]$, so we divided this interval in 256 sub-intervals labeled as $i$ and assign each gray level of the input image to the nearest bounded limit of the corresponding subinterval. This is its $z_{i}$ value required for computing the histogram bin value $p\left(z_{i}\right)$.

(4) Computing the relevance of green indices: considering that minimum uniformity is preferred against maximum, we computed the uniformity $U_{G_{k}}$ for each green image, where $\Omega=\left\{G_{k} \mid k \equiv E x G\right.$, $E x G R, C I V E, V E G\}$, firstly the uniformities were normalized in the range $[0,1]$, then the complement with respect to the unity was computed and finally again normalized in the range $[0,1]$, so we obtained the weights $w_{G_{k}}$ associated to each green image, where maximum weight is associated to minimum uniformity.

$U_{G_{k}}=\frac{1-U_{G_{k}}}{\sum_{G_{h} \in \Omega} U_{G_{h}}} ; \quad w_{G_{k}}=\frac{U_{G_{k}}}{\sum_{G_{h} \in \Omega} U_{G_{h}}}$

(5) Combining the greenness: from the four available images, we combined them by applying the following averaged rule,

$G=\sum_{\Omega} w_{G_{k}} G_{k}$

(6) Image thersholding: the greenness of the combined image $G$ was accentuated with regard to other two spectral bands ( $R$ and $B$ ) and with respect to the images which have been combined. The next goal is to isolate the green parts exploiting this improvement. As mentioned in the introduction several thresholding methods were applied for this purpose; because our approach is automatic, we look for an automatic thresholding approach, where the Otsu's method is a possible candidate which has been applied in Meyer and Camargo-Neto (2008). Nevertheless, we verified that the threshold obtained by Otsu generally tends to produce an infra-segmentation because it provides a relative high value in the histogram and many pixels are discarded as green ones. We verified that in our images it is always over the histogram mean value. This is the reason for choosing the mean instead the Otsu as threshold for binarization. Fig. 5(a) and (b) displays the binarized images obtained from $G$ by applying the histogram mean and the Otsu threshold respectively. Fig. 5(c) displays the differences between Fig. 5(a) and (b), where the white pixels correspond to green plants extracted with the mean and occluded by Otsu. In the images displayed, the values obtained for the thresholds given by the mean and Otsu are 0.2496 and 0.2706 respectively over gray values ranging in $[0,1]$, i.e. the greatest value of the second with respect to the first one makes the occlusion of the green values displayed in Fig. 5(c). Therefore, we computed the mean value of $z$ (the average gray level) as follows:

$m=\sum_{i=0}^{L-1} z_{i} p\left(z_{i}\right)$
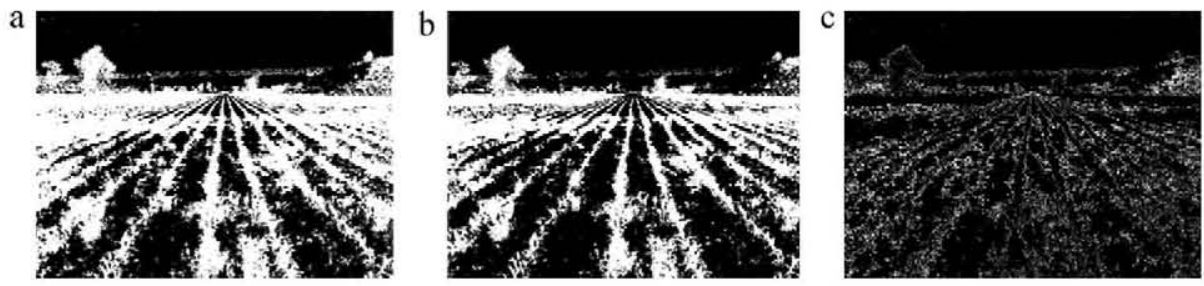

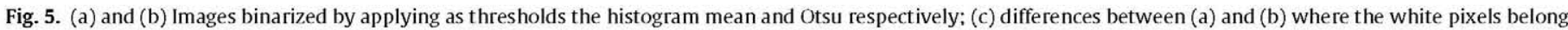
to green plants occluded in Otsu. 


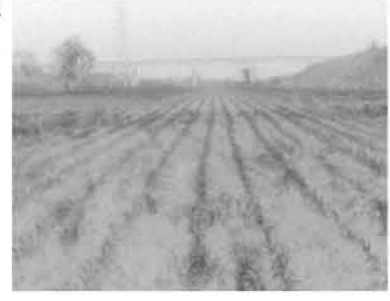

b

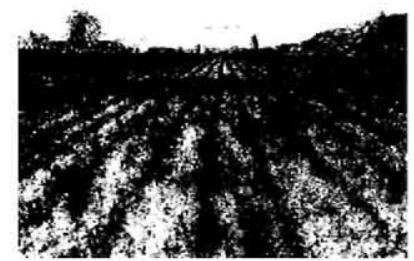

c

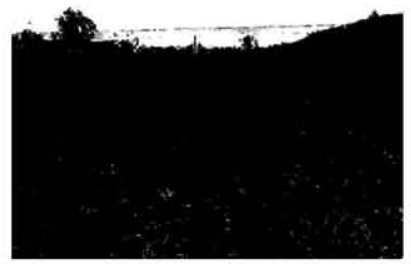

Fig. 6. (a) ExB image computed through Eq. (5); (b) binary image obtained by applying the mean histogram; (c) binary image obtained by applying the Otsu's approach.
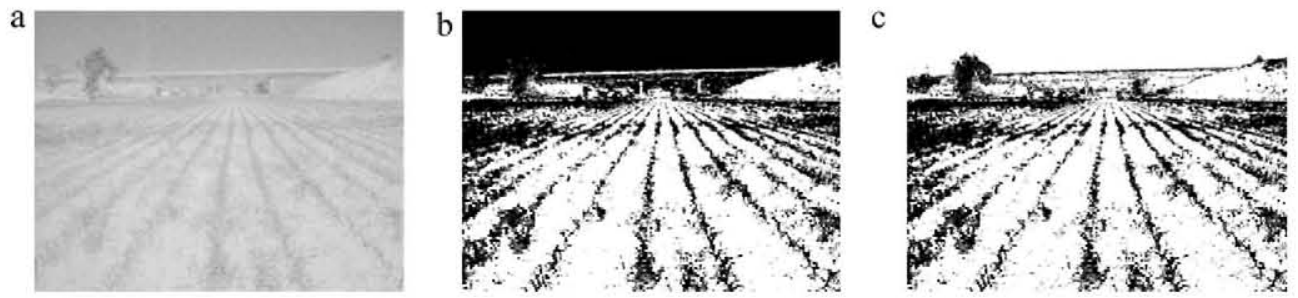

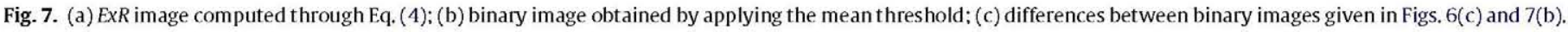

We obtained the binary image $G_{b}$ based on the mean, where those pixels with its value in $G$, Eq. (11), greater than the mean are labeled as white, i.e. they contain a high degree of greenness.

(7) Detecting the blueness: from the ExB image obtained through Eq. (5), the goal is to achieve its binarization. An important problem was identified applying the histogram mean during the extraction of the blueness. Indeed, large parts of textures belonging to the soil contain a high degree of blueness in spite of the fact that the redness should be dominant. This behavior suggests us to apply the Otsu's approach so that only those parts with a high degree of blueness can be separated retaining the rest which those causing the aforementioned problem. But, why we apply Otsu?, the reason is that it provides a value higher than the mean, as in the greenness extraction, and the sky is now well separated from the blueness belonging to the soil. The binary image representing the blueness coming from ExB is called $B_{b}$. Fig. 6, clarifies this. Indeed, in (a) the EXB image is displayed; (b) and (c) display the binary images obtained by applying the mean and Otsu thresholds. As one can see, in Fig. 6(b) an important part of soil is labeled as blue what does not occur in (c), as expected. This is a common behavior observed in the whole set of images analyzed.

(8) Detecting the redness: given the ExR image obtained from Eq. (4), as before, the goal is to achieve their binarization, taking into account that it is associated to the textures belonging to the soil. Therefore, because the soil is a relevant part in the crop fields, now we tried to retain the greatest number, as possible, of pixels that are potential candidates as textures in the soil. Therefore, like in the greenness case, we applied the mean as the best threshold value for binarization obtaining the binary image $R_{b}$. Fig. 7(a) displays the ExR image and Fig. 7(b) its binary image obtained by applying the histogram mean as threshold.

In summary, for relevant textures such as green plants and soil we applied the mean threshold with the goal of retaining the most important parts and for blueness we apply the Otsu's thresholding approach.

\subsection{Class separation: greenness and redness}

\subsubsection{Problem statement}

The next goal is the identification of different classes of greenness and also different classes of soil textures. The greenness is extracted through Eq. (11) and the green plants are well separated by the mean threshold. Nevertheless, they can still be separated in sub-classes with the aim of distinguishing different kinds of plants. When trying to extract blueness and redness we need to solve the problem pointed out before with respect to the presence of blueness in the soil. As we already know, we dealt with two kinds of images. One of the types does not contain sky and the other contains an important amount of sky. Figs. 1 and 2 are two representative types of these images respectively. If we extract blueness by applying the Otsu's method to the images containing sky this texture appears well separated from the remainder (plants and soil), as we can see in the example displayed in Fig. 6(c). Also, if we extract the greenness for the same type of images by applying the mean threshold, the soil is well separated from the remainder textures (plants and sky), Fig. 7(b). On the contrary, if blueness and redness are extracted in the images without sky, they both are associated to pixels belonging to the soil with an important overlapping on this kind of pixels. This can be observed in the images of Fig. 8(a) and (b). If we compute the difference between the binary images obtained from $E x B$ and $E x G$ the result for the images without sky is displayed in Fig. 8(c), where we can see that the ratio of white pixels is low as compared with the full image. On the contrary, if the images contain sky the difference between the binary images obtained from ExB and ExR gives the image displayed in Fig. 7(c), where one can see that the ratio between white pixels as compared to the whole
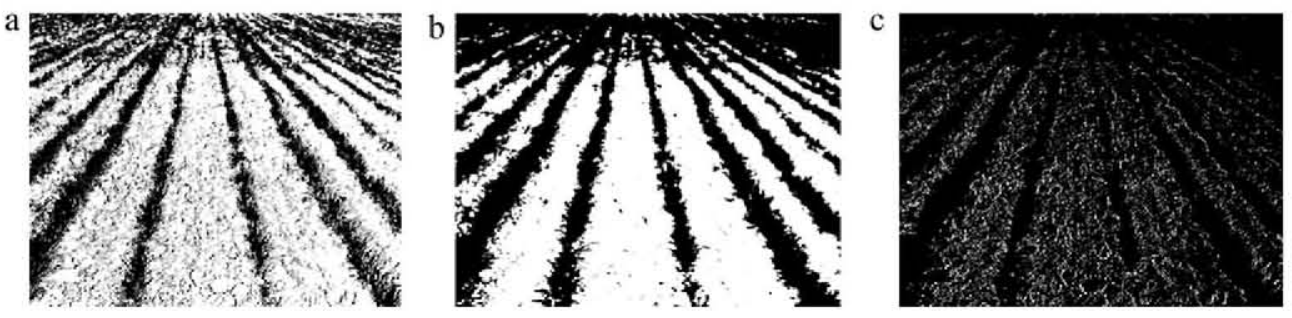

Fig. 8. Binary images obtained from the original image in Fig. 1: (a) ExB; (b) ExR; (c) difference between the binarized images ExB and ExR. 

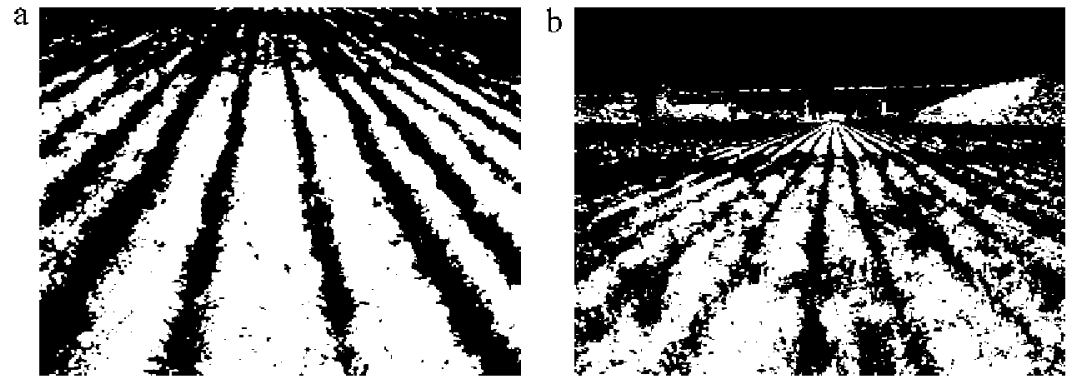

Fig. 9. White labels identifying pixels belonging to the soil: (a) for the image in Fig. 1; (b) for the image in Fig. 2.

image becomes high as compared with the above kind of images. Therefore, depending on the type of images the relation between blueness and redness differs considerably. This fact was exploited to isolate those pixels belonging to the soil independently of the type of images, i.e. with and without sky. This contributes to the design of the proposed automatic image segmentation procedure. Based on this study, we computed the following ratio:

$r=\frac{N_{w}}{N_{T}}$

where $N_{w}$ is the number of pixels with values of +1 or -1 in the image $Y$ obtained as the difference between the binary images obtained from $B_{b}$ and $R_{b} ; N_{T}$ is the total number of pixels in the image. Now, we are ready to isolate the pixels that are considered as belonging to the soil by applying the following rule-based decision making procedure:

$$
\begin{aligned}
& \text { if } r<T_{1} \text { then } \\
& S_{b}=R_{b} \wedge \neg G_{b} \\
& \text { else } \\
& \quad S_{b}=R_{b} \wedge \neg G_{b} \wedge \neg B_{b}
\end{aligned}
$$

where the symbols $\wedge$ and $\neg$ are the logical "and" and "not" operations over the binary images; $S_{b}$ is the final binary image containing the soil pixels as white labels. The explanation of the rule in Eq. (14) is as follows. When $r$ in Eq. (13) is low $N_{w}$ is also low as compared to $N_{T}$, this means that there are a lot of pixels coming from the blueness that match with redness, all belonging to the soil and the image does not contain sky. Therefore, for extracting the redness, we only need to exclude from the whole image the greenness. On the contrary, if $r$ in Eq. (13) is high $N_{w}$ is also high and the image contains sky, therefore, we now excluded from the whole image both greenness and blueness. The final redness is extracted in the binary image $S_{b}$ as white labels representing the pixels belonging to the soil. The threshold $T_{1}$ is easy to fix, indeed because $r$ generally tends towards 1 or 0 depending on the type of images, i.e. with and without sky respectively, by setting it to the intermediate value of 0.5 it suffices. Fig. 9(a) and (b) displays those pixels belonging to the soil extracted from the original images in Figs. 1 and 2 respectively.

\subsubsection{Classification based on fuzzy clustering}

Meyer et al. (2004) applied fuzzy clustering for plant silhouette identification and the fuzzification is carried out directly to $R$, $G, B$ or ExG and ExR. Unlike the Meyer et al. (2004) approach, we have two available main binary images, $G_{b}$ and $S_{b}$ representing the degrees of greenness and redness respectively. Based on them, we extracted two sets of samples from the original image, identified as $X_{G}$ and $X_{S}$. The first set $X_{G}$ contains samples representing the pixels in the original image which have been identified as green pixels in $G_{b}$ and $X_{S}$ contains those pixels representing the soil. Without lost of generality, we represented those sets under the generic denomination $X$ with $n$ training samples, i.e., $X=\left\{x_{1}, x_{2}, \ldots, x_{n}\right\} \in \Re^{d}$, where $d$ is the data dimensionality. Each sample vector $x_{i}$ represents an image pixel, where its components are the three RGB spectral components of that pixel at the image location $(x, y)$ given by $G_{b}$ and $S_{b}$. This means that in our experiments the data dimensionality is $d=3$. Each sample is to be assigned to a given cluster $w_{j}$, where the number of possible clusters is $c$, i.e., $j=1,2, \ldots, c$.

The samples in $X$ are to be classified based on the well-known fuzzy clustering approach that receives the input training samples $\boldsymbol{x}_{i}$ and establishes a partition, assuming the number of clusters $c$ is known. The process computes for each $\boldsymbol{x}_{i}$ at the iteration $t$, its degree of membership in the cluster $w_{j}\left(\mu_{i}^{j}\right)$ and updates the cluster centers $v_{j}$ as follows (Zimmermann, 1991):

$$
\begin{aligned}
\mu_{i}^{j}(t+1) & =\frac{1}{\sum_{r=1}^{c}\left(d_{i j}(t) / d_{i r}(t)\right)^{2 /(b-1)}} ; \\
v_{j}(t+1) & =\frac{\sum_{i=1}^{n}\left[\mu_{i}^{j}(t)\right]^{b} \boldsymbol{x}_{i}}{\sum_{i=1}^{n}\left[\mu_{i}^{j}(t)\right]^{b}}
\end{aligned}
$$

$d_{i j}^{2} \equiv d^{2}\left(\boldsymbol{x}_{i}, v_{j}\right)$ is the squared Euclidean distance. The number $b$ is called the exponential weight (Bezdek, 1981; Duda et al., 2000), $b>1$. The stopping criterion of the iteration process is achieved when $\left\|\mu_{i}^{j}(t+1)-\mu_{i}^{j}(t)\right\|<\varepsilon \quad \forall i j$ or a number $t_{\max }$ of iterations is reached.

The method requires the initialization of the cluster centers, so that Eq. (15) can be applied at the iteration $t=1$. For this purpose, we applied the pseudorandom procedure described in Balasko et al. (2008):

(1) Perform a linear transform $Y=f(X)$ of the training sample values so that they range in the interval $[0,1]$.

(2) Initialize $v=2 D \overline{\boldsymbol{M}} \circ \boldsymbol{R}+D \overline{\boldsymbol{m}}$, where $\overline{\boldsymbol{m}}$ is the mean vector for the transformed training samples values in $Y$ and $\bar{M}=\max (a b s(Y-$ $\overline{\mathbf{m}})$ ), both of size $1 \times d ; D=[1 \ldots 1]^{T}$ with size $c \times 1 ; \boldsymbol{R}$ is a $c \times d$ matrix of random numbers in $[0,1]$; the operation $\circ$ denotes the element by element multiplication.

(3) Merging process: once the fuzzy clustering process is carried out, a partition of the input training samples is obtained, where each cluster $\boldsymbol{w}_{j}$ has associated its centre $\boldsymbol{v}_{j}$. Now if two clusters are considered close among them, they are fused in a unique cluster. The criterion to fuse the clusters is established based on the proximity of the centers, so given the two centers $v_{i}$ and $v_{j}$ their respective clusters are fused if $d\left(\boldsymbol{v}_{i}, \boldsymbol{v}_{j}\right)<T_{2}$; where $d$ is the Euclidean norm and $T_{2}$ is a threshold set to the $20 \%$ of the maximum distance possible between the samples in the cluster, i.e. $T_{2}=0.2 \sqrt{3}$; this value was obtained assuming that the spectral pixel values range from $(0,0,0)$ (black) to $(1,1,1)$ (white) in the RGB color space and its maximum distance is the length of the main diagonal in the cube representing this space. When two clusters are fused, we compute its new cluster center as the average between all samples belonging to both clusters that have been fused. 


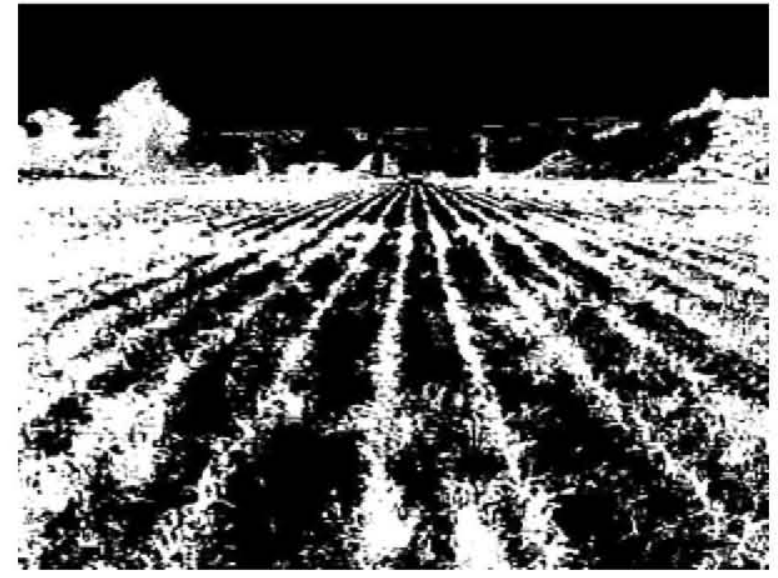

Fig. 10. Ground truth image for greenness from image in Fig. 2.

\section{Results}

The proposed strategy consists of two main processes, namely: (a) extraction of greenness, redness and blueness; (b) identification of different types of greenness and redness.

The extraction of greenness was based on a new approach, which combines the information provided by the following four approaches that also compute greenness separately, i.e. ExG, ExGR, CIVE and VEG. Therefore, we tested the performance of the proposed combined approach for greenness against these methods separately. This was carried out based on a set of ground-truth images which are built as follows: (1) given the original image in the RGB color space, we extracted all pixels where the Green component is greater than the Red and Blue ones simultaneously at least in a $10 \%$. This allows us to obtain an important part of green plants, which are labeled as white; (2) also from the original image we extracted those parts where the Red and Blue components are dominant, i.e. they overpass at least in a value of $10 \%$ the other two. This allows extracting the Red and Blue parts with a certain degree of confidence, being labeled as black; (3) the pixels that still were not labeled were manually touched up and labeled according to the human expert criterion by comparing them with the original image. Fig. 10 displays a ground-truth image obtained from the original image given in Fig. 2.

We used in our experiments 240 original images acquired as described in Section 2.1, where 190 are images containing sky and the remainder ones, i.e. 50 , without sky. About the $10 \%$ of each group were acquired under poor illumination conditions. We built 60 ground-truth images from the set containing sky and 15 from the set without sky; we built images acquired under different illumination conditions. We verified that the method behaves similarly for both, i.e. for images with low and high lighting conditions, there-
Table 1

Percentage of error averaged over the set of images with ground truth.

\begin{tabular}{llllll}
\hline & EXG & EXGR & CIVE & VEG & COM \\
\hline \% of error for greenness & 11.19 & 10.71 & 10.37 & 18.23 & 8.31 \\
\hline
\end{tabular}

fore it is unnecessary a distinction among them when testing the proposed method.

Table 1 displays the percentage of error averaged over the number of images tested based on the corresponding ground truth for ExG, ExGR, CIVE and VEG against our proposed combined approach (COM).

For each image which is combined we obtained four weights through Eq. (10), which are applied in Eq. (11) for computing the final greenness. The averaged values over the set of the 240 images are finally the following: $w_{G_{E X C}}=0.25, w_{G_{E X C R}}=0.30, w_{G_{C N E}}=0.33$ and $w_{G V G}=0.12$. As we can see there is a direct relation between these averaged weights and the percentage of error displayed in Table 1. Based on the weights, we can see that the most relevant method is CIVE, where its contribution to the combination was on average of 0.33 , which is the greatest and simultaneously it gives the less percentage of error among the remainder ones, i.e. ExG, ExGR and VEG. The less relevant was VEG with 0.12 , giving the greatest percentage of error. In this last case perhaps its behavior could be due to the parameter $a$ involved in Eq. (8), although after different experiments, we did not find a clear relation between this behavior and its relevance.

In summary, with respect to the greenness the proposed strategy outperforms the simple ones in terms of percentage of error. Moreover it establishes an automatic mechanism of combination. Its main drawback is the increase of the computational time, where the time for computing the greenness is approximately increased in a rate of 1 to 4 because it needs the computation of four indices instead of the unique computation required individually.

The averaged percentage of errors for redness and blueness are respectively 10.87 and 10.91 with a similar behaviour to the EXGR in greenness and similar among them, this is because EXR and EXB are both computed similarly through Eqs. (4) and (5) where only the normalized $r$ and $b$ spectral components are interchanged.

We verified over the set of images analyzed that in average, the threshold obtained by Otsu is greater than the one obtained by the histogram mean about a $16 \%$ and a $12 \%$ in the images containing sky and without sky respectively.

Fig. 11(a) and (b) displays the results obtained after applying the fuzzy clustering approach to the image in Fig. 2. The number of clusters used for this classifier was initially set to $c=3$. In (a) the results obtained for green plants, where we can see that three classes of green plants were distinguished, i.e. no fusion of clusters was carried out. Indeed, from the point of view of a human observer it is easy to see that there are three classes, where one of the classes is associated to a dark green tonality belonging to the trees and shades in the furrows. The other two correspond to two different
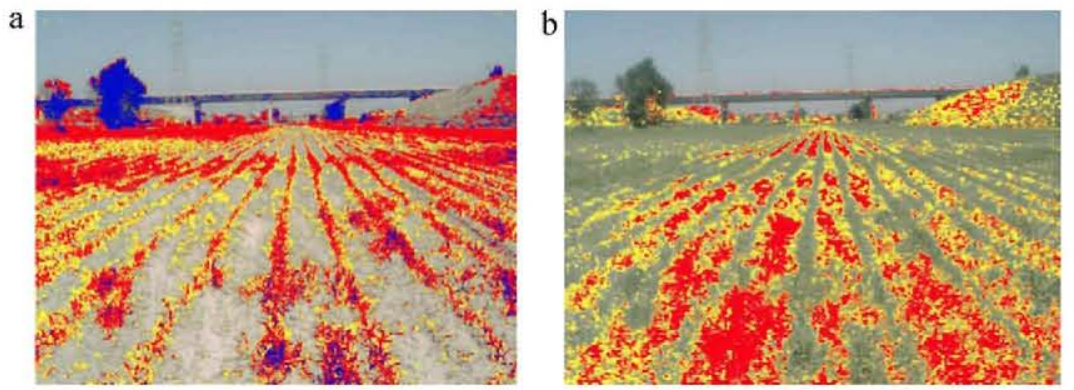

Fig. 11. Classification results for the image in Fig. 2: (a) three different classes of green plants; (b) two different classes in the soil. 

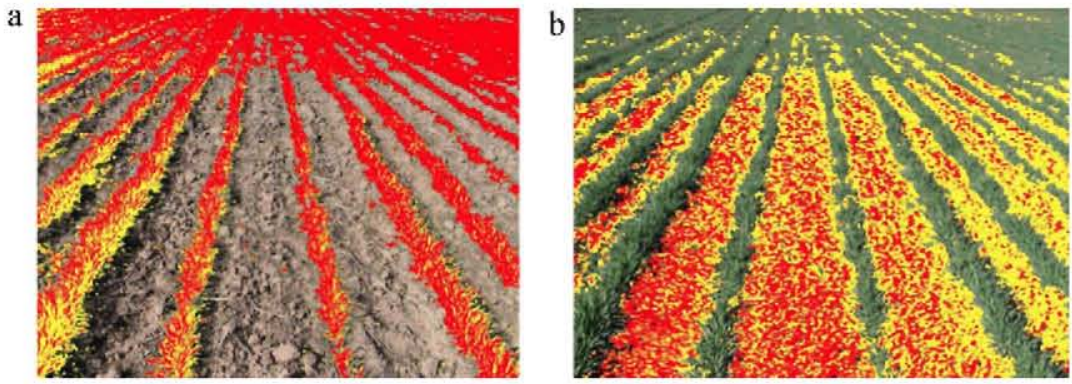

Fig. 12. Classification results for the image in Fig. 1: (a) two different classes of green plants; (b) two different classes in the soil.

tonalities in the plants. On the contrary in Fig. 11(b) a fusion process was carried out and two classes were finally obtained, which allows distinguishing two types of textures.

As before, Fig. 12(a) and (b) displays the results obtained after applying the fuzzy classifier to the original image in Fig. 1 with $c=3$. In (a) the results obtained for green plants, here the decision made is the fusion of two clusters, this is because the greenness is very homogeneous and only the shades are distinguished as dark plants. This will allow to determine the position of the sun based on the shades; indeed because the main density of shades are detected in the left part of the image, we can infer that the illumination coming from the sun arrives by the left part of the image, that perhaps could be of interest in subsequent applications.

In the proposed approach we applied the fuzzy clustering as a method for discriminate different green and soil textures once they were isolated, this means that the samples for training are supplied automatically without the supervisor intervention. Moreover, because the greenness and redness identification is not affected by the lighting conditions, the learning does not require samples with these differences. This represents the main advantage with respect to the general learning strategies such that those referenced in the introduction.

\section{Conclusions}

We proposed a new strategy for automatic image segmentation from crops of cereal and corn, where the plants and the soil are identified. The goal is to improve the performance of some existing segmentation processes, mainly in the extraction of the green parts containing the crops and even the weeds. This was achieved by applying a combined strategy based on the information of the images through the uniformity. We verified that the combined approach obtains better results that the methods used separately. The strategy was valid for two types of images, those containing sky and those without it. Both were processed with the purpose of site specific agriculture treatments. The first ones contain a broadest field of view with the purpose of identifying possible structures which could be useful during autonomous navigation of a vehicle equipped with the imaging system. We also designed a new automatic strategy for discriminating different textures in the part of green plants and also in the soil, which are also useful for specific site treatments and to recover important information contained in the field for future applications and treatments, perhaps during the next crop cycles. The method proved its effectiveness under different illumination conditions in outdoor environments, with a high degree of difficulty. Future improvements could be considered in this line trying to minimize the risk of the illumination by applying techniques such as homomorphic filtering, which is technique tested in outdoor environments with a high degree of effectiveness (Pajares et al., 2005). The proposed method has been tested in barley and corn fields, which are crops containing green plants and visible soil parts with dominant red tonalities and perhaps sky with dominant blueness. Therefore, it can be applied to any type of crops with such characteristics being of particular interest the fields of cereals such as wheat or rye.

Perhaps the increase of computational time in the combined strategy for computing the greenness with respect to the individual methods can be reduced in the future under parallel architectures.

\section{Acknowledgements}

The authors wish to acknowledge to the projects AGL-200804670-C03-02, DPI2009-14552-C02-01 projects, supported by the Ministerio de Educación y Ciencia of Spain within the Plan Nacional de $I+D+i$. Also to the EU which has granted the project in the Theme NMP-2009-3.4-1 (Automation and robotics for sustainable crop and forestry management) with number 245986. Also this document was prepared with economical support of the European Community, the European Union and CONACYT under grant FONCICYT 93829. The content of this document is an exclusive responsibility of the University Complutense, and cannot be considered as the position of the European Community. The authors wish to acknowledge to the Council of Education of the Autonomous Community of Madrid and the Social European Fund for the research contract with the fourth author. Finally, the authors are grateful to the referees for their suggestions and constructive criticism of the original version of this paper.

\section{References}

Balasko, B., Abonyi, J., Feil, B., 2008. Fuzzy Clustering and Data Analysis Toolbox for Use with Matlab. Veszprem University, Hungary, available on-line from the web site: http://www.fmt.vein.hu/softcomp/fclusttoolbox/FuzzyClústeringToolbox. pdf.

Bezdek, J.C., 1981. Pattern Recognition with Fuzzy Objective Function Algorithms. Kluwer, Plenum Press, New York.

Burgos-Artizzu, X.P., Ribeiro, A., Tellaeche, A, Pajares, G., Fernández-Ouintanilla, C., 2009. Improving weed pressure assessment using digital images from an experience based reasoning approach. Computers and Elect ronics in Agriculture $65,176-185$.

Davies, G., Casady, W., Massey, R., 1998. Precision agriculture: an introduction. In: Water Quality Focus Guide, WQ450, available on-line: http://extension. missouri.edu/explorepdf/envqual/wq0450.pdf.

Duda, R.O., Hart, P.E., Stork, D.S., 2000. Pattern Classification. Wiley.

Gebhardt, S., Schellberg, J., Lock, R., Kaühbauch, W.A., 2006. Identification of broadleaved dock (Rumex obtusifolius L.) on grassland by means of digital image processing. Precision Agriculture 7 (3), 165-178.

Gebhardt, S., Kaühbauch, W.A., 2007. A new algorithm for automatic Rumex obtusifolius detection in digital image using colour and texture features and the influence of image resolution. Precision Agriculture 8 (1), 1-13.

Gée, Ch., Bossu, J., Jones, G., Truchetet, F., 2008. Crop/weed discrimination in perspective agronomic images. Computers and Electronics in Agriculture 60, 49-59.

Gonzalez, R.C., Woods, R.E., 2008. Digital Image Processing. Prentice-Hall, Upper Saddle River, NY.

Gonzalez, R.C., Woods, R.E., Eddins, S.L, 2004. Digital Image Processing using Matlab. Prentice-Hall, Upper Saddle River, NY.

Hague, T., Tillet, N., Wheeler, H., 2006. Automated crop and weed monitoring in widely spaced cereals. Precision Agriculture 1 (1), 95-113.

Kataoka, T., et al., 2003. Crop growth estimation system using machine vision. In: The 2003 IEEE/ASME International Conference on Advanced Intelligent Mechatronics. 
Kirk, K, Andersen, H.J., Thomsen, A.G., Jørgensen, J.R., 2009. Estimation of leaf area index in ceral crops using red-green images. Biosystems Engineering 104, $308-317$.

Ling, P.P., Ruzhitsky, V.N., 1996. Machine vision techniques for measuring the canopy of tomato seedling. Journal Agricultural Engineering Research 65 (2), 85-95.

Luscier, J.D., Thompson, W.L., Wilson, J.M., Gorham, B.E., Dragut, L.D., 2006. Using digital photographs and object-based image analysis to estimate percent ground cover in vegetation plots. Frontiers in Ecology and the Environment 4 (8), $408-413$.

Meyer, G.E., Hindman, T.W., Lakshmi, K., 1998. Machine Vision Detection Parameters for Plant Species Identification. SPIE, Bellingham, WA

Meyer, G.E., Camargo-Neto, J., Jones, D.D., Hindman, T.W., 2004. Intensified fuzzy clusters for classifying plant, soil, and residue regions of interest from color images. Computers and Electronics in Agriculture 42, 161-180.

Meyer, G.E., Camargo-Neto, J., 2008. Verification of color vegetation indices for automated crop imaging applications. Computers and Elect ronics in Agriculture 63, $282-293$.

Neto, J.C., 2004. A Combined Statistical-Soft Computing Approach for Classification and Mapping Weed Species in Minimum Tillage Systems. University of Nebraska, Lincoln, NE.

Onyango, C.M., Marchant, J.A., 2003. Segmentation of row crop plants from weeds using colour and morphology. Computers and Electronics in Agriculture 39, $141-155$.

Otsu, N., 1979. A threshold selection method from gray-level histogram. IEEE Trans actions on System Man and Cybernetics 9, 62-66.

Pajares, G., Ruz, J.J., Cruz, J.M., 2005. Performance analysis Performance Analysis of Homomorphic Systems for Image Change Detection. In: Marques, J.S., de la Blanca, N.P., Pins, P. (Eds.), Pattern Recognition and Image Analysis, vol. 3522. Springer-Verlag, Berlín, pp. 563-570.

Reid, J.F., Searcy, S.W., 1987. Vision-based guidance of an agricultural tractor. IEEE Control Systems 7 (12), 39-43.
Ribeiro, A, Fernández-Quintanilla, C, Barroso, I. García-Alegre, M. 2005 Development of an image analysis system for estimation of weed. In: Proceedings of the 5th European Conference On Precision Agriculture (5ECPA), pp. $169-174$.

Ruiz-Ruiz, G., Gómez-Gil, J., Navas-Gracia, L.M., 2009. Testing different color spaces based on hue for the environmentally adaptive segmentation algorithm (EASA). Computers and Electronics in Agriculture 68, 88-96.

Shrestha, D.S., Steward, B.L., Birrell, S.J., 2004. Video processing for early stage maize plant detection. Biosystems in Engineering 89 (2), 119-129.

Tellaeche, A., Burgos-Artizzu, X., Pajares, G., Ribeiro, A., Fernández-Quintanilla, C., 2008a. A new vision-based approach to differential spraying in precision agriculture. Computers and Electronics in Agriculture 60 (2), 144-155.

Tellaeche, A., Burgos-Artizzu, X.P., Pajares, G., Ribeiro, A., 2008b. A vision-based method for weeds identification through the Bayesian decision theory. Pattern Recognition 41, 521-530.

The Mathworks 2010. http://www.mathworks.com/

Tian, Slaughter, 1998. Environmentally adaptive segmentation algorithm for outdoor image segmentation. Computers and Electronics in Agriculture 21 $153-168$.

Woebbecke, D.M., Meyer, G.E., von Bargen, K., Mortensen, D.A., 1995. Shape features for identifying young weeds using image analysis. Transactions on American Society of Agricultural Engineering 38 (1), 271-281.

Zheng, L., Zhang, J., y Wang, Q, 2009. Mean-shift-based color segmentation of images containing green vegetation. Computers and Electronics in Agriculture $65,93-98$.

Zheng, L., Shi, D., Zhang, J., 2010. Segmentation of green vegetation of crop canopy images based on mean shift and Fisher linear discriminate. Pattern Recognition Letters, doi:10.1016/j.patrec.2010.01.016.

Zimmermann, H.J., 1991. Fuzzy Set Theory and its Applications. Kluwer Academic Publishers, Norwell. 